\section{Use of an over-the-scope clip and a colonoscope for complete hemostasis of a duodenal diverticular bleed}

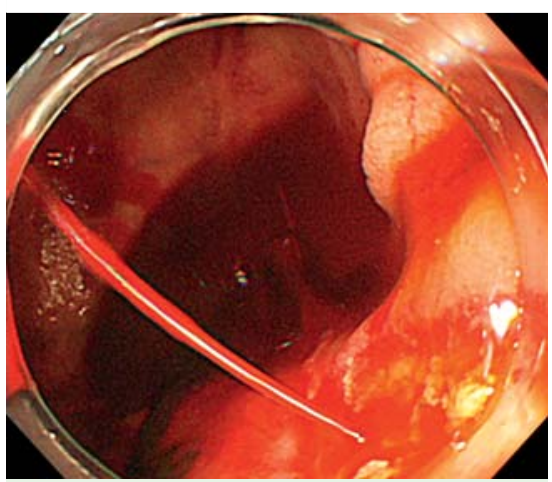

Fig. 1 Endoscopic view of spurting bleeding from a Dieulafoy lesion at the edge of a large diverticulum in the third portion of the duodenum in an 81-year-old man.

Duodenal diverticular bleeding has traditionally required invasive surgical repair. With improvements in endoscopic devices, endoscopic treatment has become the main therapy for such bleeding $[1,2]$. However, the question of which endoscopic devices should be used is still controversial because of complications and recurrent bleeding after treatment [3]. A newly developed device, the overthe-scope clip (OTSC) [4], has been reported to be useful for treatment of bleeding gastrointestinal lesions that are resistant to conventional therapy [5]. Here we report the first case of complete hemostasis using an OTSC system and a colonoscope for bleeding from a Dieulafoy lesion that was located in a duodenal diverticulum and was resistant to conventional therapy. An 81-year-old man presented with tarry stool and severe anemia. Upper gastrointestinal endoscopy failed to detect the bleeding site. Re-bleeding occurred during a capsule endoscopy. Informed consent was obtained from the patient before the following treatment was undertaken. Because fresh blood had been found from the deep second portion of the duodenum toward the anal side, a colonoscope with a waterjet function (PCF-Q260AI; Olympus, Tokyo, Japan) was introduced. This contributed to clear identification of a Dieulafoy lesion at the edge of a large diverticulum in the third portion of the duodenum. Endoscopic hemostasis using hemostatic forceps (Coagrasper, FD-411UR; Olympus) only diminished the blood flow from spurting ( Fig. 1) to oozing. Because of the risk of perforation when using coagulation in the thin duodenal wall, an OTSC system (Ovesco Endoscopy, Tübingen, Germany) was selected as the next step. The entire defect of the bleeding ulcer was successfully closed using the OTSC

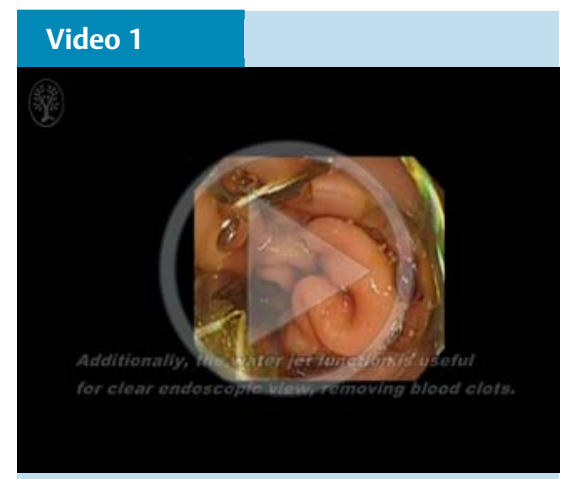

First case of complete hemostasis using an over-the-scope clip (OTSC) and a colonoscope for bleeding from a Dieulafoy lesion that was located in a duodenal diverticulum and resistant to conventional therapy. The use of a colonoscope with a waterjet function helped to clearly identify the Dieulafoy lesion at the edge of a large diverticulum in the third portion of the duodenum. First, endoscopic hemostasis using hemostatic forceps fails to achieve complete hemostasis; oozing bleeding from the exposed vessel can be seen. The use of the colonoscope helps in reaching the third portion of the duodenum. Additionally, the waterjet is useful for obtaining a clear endoscopic view by removing blood clots. Second, an OTSC system is introduced. The bleeding site is aspirated into the application cap, then an OTSC is deployed. The entire defect of the bleeding ulcer is successfully closed, resulting in complete hemostasis. There is no recurrence of bleeding and no associated complications during the 6-month follow-up period.

as shown in the schema ( $\bullet$ Fig. 2 a-c), resulting in complete hemostasis ( $\bullet$ Fig.3, Video 1).
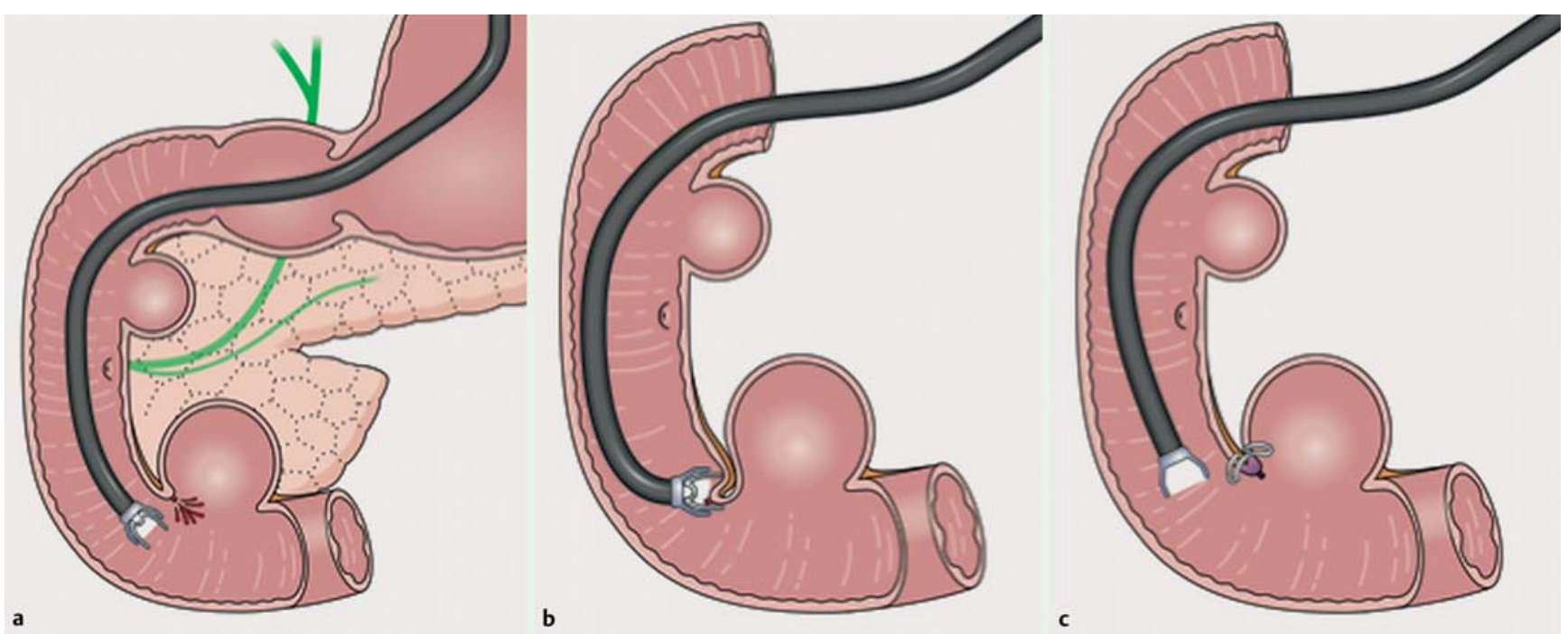

Fig. 2 Schema illustrating placement of an over-the-scope clip (OTSC) to treat a bleeding Dieulafoy lesion in a duodenal diverticulum. a Use of colonoscope to detect the bleeding site in a duodenal diverticulum. b Suction of the bleeding site into the application cap of the OTSC system. $\mathbf{c}$ Complete closure of the entire defect of the bleeding ulcer by the OTSC. 


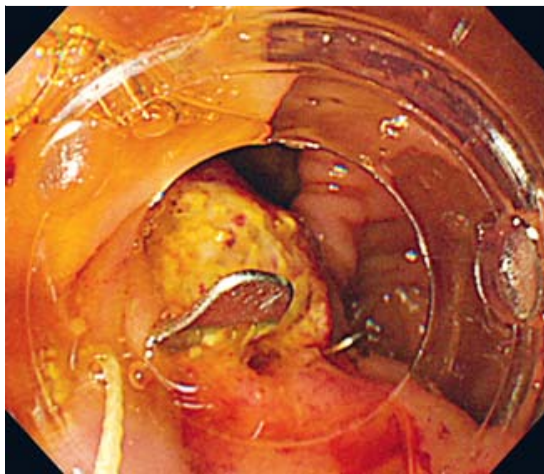

Fig. 3 Endoscopic view showing complete hemostasis by successful placement of an overthe-scope clip (OTSC).

Computed tomography (CT) 7 days later showed successful placement of an OTSC in the duodenal diverticulum ( Fig.4). Our patient had an excellent outcome with no recurrence of bleeding or associated complications over the 6-month follow-up period. This case demonstrates that OTSC deployment is an effective and secure treatment option for the management of duodenal diverticular bleeding.

Endoscopy_UCTN_Code_TTT_1AO_2AD

Competing interests: None

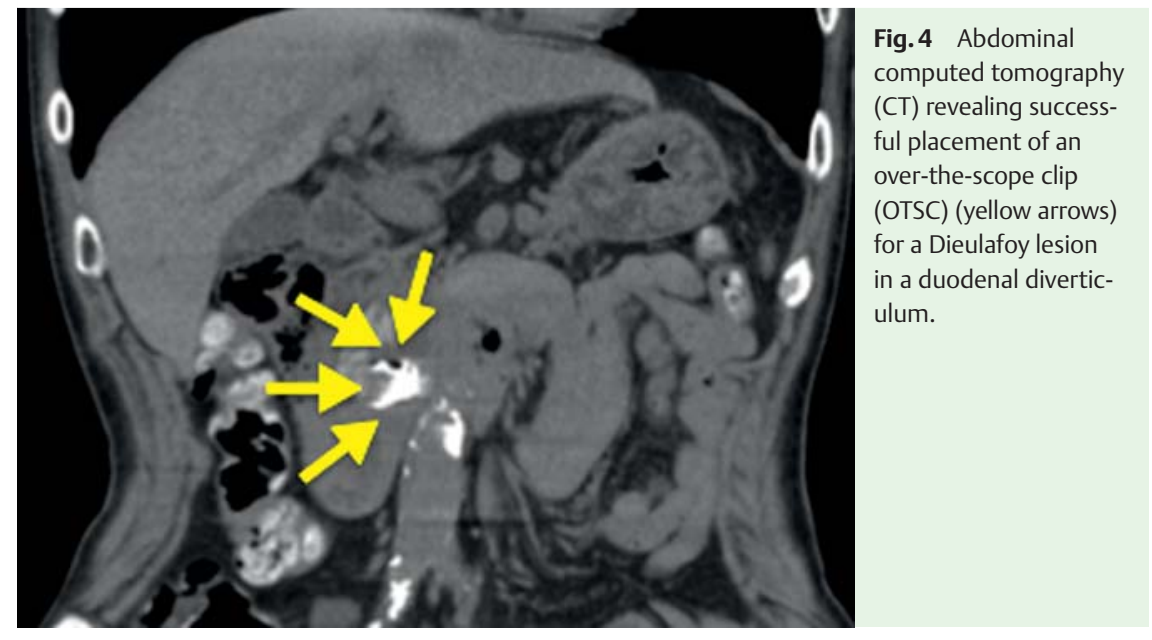

Shintaro Fujihara, Hirohito Mori, Hideki Kobara, Noriko Nishiyama, Maki Ayaki, Toshiaki Nakatsu, Tsutomu Masaki

Department of Gastroenterology and Neurology, Faculty of Medicine, Kagawa University, Japan

\section{References}

1 Kachi M, Fujii M, Tateiwa S et al. Endoscopic injection therapy for the treatment of duodenal diverticulum bleeding. J Gastroenterol Hepatol 2002; 17: 1241 - 1242

2 Moyer MT, Bethards DM. Identification and treatment of a duodenal diverticular hemorrhage: an illustration of the usefulness of the jumbo-channel therapeutic endoscope. Endoscopy 2009; 41: E230 -E231

3 Chen YY, Yen HH, Soon MS. Impact of endoscopy in the management of duodenal diverticular bleeding: experience of a single medical center and a review of recent literature. Gastrointest Endosc 2007; 66: 831 -835

4 Kirschniak A, Kratt T, Königsrainer A et al. A new endoscopic over-the-scope clip system for treatment of lesions and bleeding in the
GI tract: first clinical experiences. Gastrointest Endosc 2007; 66: $162-167$

5 Nishiyama $\mathrm{N}$, Mori $\mathrm{H}$, Kobara $\mathrm{H}$ et al. Efficacy and safety of over-the-scope clip: including complications after endoscopic submucosal dissection. World J Gastroenterol 2013; 19: $2752-2760$

Bibliography

Dol http://dx.doi.org/

10.1055/s-0034-1392212

Endoscopy 2015; 47: E236-E237

(c) Georg Thieme Verlag KG

Stuttgart · New York

ISSN 0013-726X

\section{Corresponding author}

\section{Shintaro Fujihara, MD}

Department of Gastroenterology and Neurology, Faculty of Medicine

Kagawa University

1750-1 Ikenobe, Miki, Kita

Kagawa 761-0793

Japan

Fax: +81-87-8912158

joshin@med.kagawa-u.ac.jp 